\author{
Entela Shkembi ${ }^{1 *}$, Evin Dani ${ }^{2}$, Nicola \\ Daniele ${ }^{3}$, Francesco Zinno ${ }^{3}$ and Gallo \\ Emiliano Omar ${ }^{4}$ \\ ${ }^{1}$ Resposabile Seminology Laboratory at Medical \\ Center, Dani Andrology, Italy \\ ${ }^{2}$ Andrologists treat male infertility at Medical Center \\ - Dani Andrology, Tirane, Albania, Italy \\ ${ }^{3}$ Department of Molecular Biology and \\ Biotechnology, Cryo Lab S.r.l. University of Rome \\ Tor Vergata Italy \\ ${ }^{4}$ Specialist of Gynecology and Obstetrics at University \\ Hospital Sassari, Italy \\ Dates: Received: 03 May, 2016; Accepted: 01 \\ June, 2016; Published: 03 June, 2016 \\ *Corresponding author: Dr. Entela Shkëmbi, \\ Resposabile Seminology Laboratory at Medical \\ Center, Dani Andrology, Rruga Qemal Stafa, \\ mbrapa Prokurorise se pergjithshme, Pall ENI nr \\ 13, Tirane, Albania, Tel: +355696674345; E-mail : \\ e.shkembi86@yahoo.com \\ www.peertechz.com
}

\section{Case Report \\ How important is the Morphology of the Semen Analysis?}

\section{Introduction}

The eminent characters like the quantity of spermatozoa with a regular figure, generally calculated by a plain semen examination, can settle only some of the trigger issues. The connection between sperms deficiency and following pathologies can be further determined with processes to measure methodically the cytomorphological performing of a man's semen -

- acute prostatitis containing coiled sperm;

- tapered sperm cells characterized varicocele,

- Instability of epididymal function typified by asthenozoospermia.

The historical WHO methodologies for morphological assessment of men's semen, based on population studies that took the average morphology of groups of men who were able to get their partner pregnant within the last are basically:

The Publication Manual: Initially adopted since the 1980 and 1987 [1], in World Health Organization (WHO) and only partially accepted in the 1992 WHO.

The Kruger Approach (strict criteria approach). Introduced by Roelof Menkveld in 1987 [2], showed in detail in 1990 [3] and in the WHO'92 this criteria were partially accepted. The IV edition (World Health Organization, 1999) Kruger criteria became the advocated assessment technique and only in the $\mathrm{V}$ edition of World Health Organization 2010 [4-6], were established as the standard method.

Kruger strict criteria focus upon the function of usual emerging sperm, this metodologiacal identification all morphologically atypical spermatozoa (Figure 1).

For routine work, a staining method such as the modified Giemsa procedure or hematoxylin and eosin staining. Thin slices were then made from the paraffin samples, immobilized on slides, dewaxed and subsequently stained, usually with hemotoxylin and eosin, and sealed with a cover slip (Figure 2).

\section{Staining procedure}

- Stain rehydrated sections in Hematoxylin solution for 20-40 minutes.

- Wash in tap water for 1-5 minutes, until sections turn blue ("bluing").

- Differentiate sections in 70\% ethanol-containing $1 \% \mathrm{HCl}-$ for 5 seconds. This removes excess dye, allowing nuclear details to emerge.

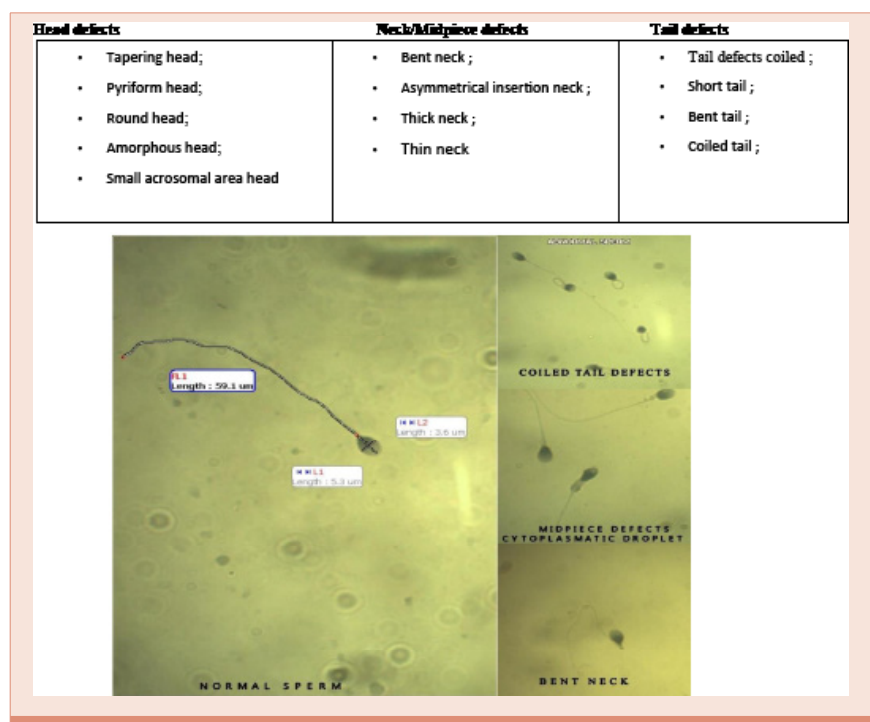

Figure 1: Sperm Morphology (Medical center-Dani Andrology. 
- Wash 1-5 minutes in tap water until blue.

- Stain in Eosin solution for 10 minutes.

- Wash 1-5 minutes in tap water.

- Dehydrate, clear and mount.

For a finest and high-quality apparition discoloration of spermatozoa are suggested with Gimsa, Shorr or Papanicolaou blot. In this examination we should assess two hundred spermatozoa apiece slide to lessen sampling inaccuracy. A 100X oil captivation intent should be used (1000X intensification) to an additional evidently identify sperm detail. Several laboratories often discard the morphological investigation of the semen. The obtained results of the percentage of morphologically normal spermatozoa, expressed as \% typical, this is the grounds why from fifth version of World Health Organization 2010 was accepted a fresh standard for the indication values for the so called apparently typical spermatozoa. This merit was abridged up to $4 \%$ of morphologically regular spermatozoa, in semen examination carried out according to uniformed methodologies [7]. At this sight, a morphologically common spermatozoa, according to Kruger [8]. In some men persistent sperm morphology abnormalities are present as a consequence of genetic factors, such as the failure of the acrosome to develop, causing the "small round-head defect" or "globozoospermia" defect. Lack of sperm acrosome is defined as an incongruity in the arrangement. They are also devoid of the post-acrosomal casing and nuclear ring. Type II has found that the encircling outline was created by a globular nucleus with an missing acrosome.

\section{Case Report}

\section{Case 1}

The first patient presented was 37 years-old men was merried of 26-year-old woman. A motive of the presentation in our clinic was a slight discomfort in the testicles. By andrology control he result orchitis diagnoses. Orchitis is an inflammation of the testicles. It can be caused by either bacteria or a virus. Both testicles may be affected by orchitis at the same time. We have recommended to do also as seminal fluid, even as he could not to have children. He had effectuated sessions and semen examination 22 times in a 6 dissimilar laboratories. His semen concentration has been reported as of 43.0 (mil/ml); progressive sperm motility by $54 \%-68 \%$ and a standard morphology (Figure 3).

And the reproductive hormones;

Follicle Stimulating Hormone (FSH): $2.84 \mathrm{mIU} / \mathrm{mL}$ (0.60-12.00)

Luteinizing Hormone (LH): $1.15 \mathrm{mIU} / \mathrm{mL}$ (0.60-12.00)

Testosterone: $5.2 \mathrm{ng} / \mathrm{mL}(2.40-8.70)$

Prolactin (Prl): 11.2 ng/mL (3.5-20.0)

His wife she had regular ovulatory cycles, normal hormonal profile and normal hysteroscopy. The woman was stimulated using long protocol with $3.75 \mathrm{mg}$ Decapeptyl and 225 IU Menogon for 11 days. At these time we keeping under control Estradiol E2 and FSH. From a total of 37 oocytes only 9 have arrived at a fertilization rate.
The woman failed to conceive in this cycle and a subsequent thaw embryo transfer cycle also failed.

\section{Case 2}

The second patient B.I a 32-year-old-man, he arrived in our clinical, with a narrative of two marriages for seven and four years each. With his first wife 25-year-old woman, the marriage had lasted seven years. And during these years he tried to have daughters, but this was not the case. The much desire to have a son, he thought that his wife could not have children, because the semen analysis results came out normal. Concentration of $72(\mathrm{mil} / \mathrm{ml})$; progressive sperm motility of $64 \%$ and a normal morphology. He had effectuated consultations and semen analysis in a 4 different laboratories. The reproductive hormones was normally same at the first case. In May of 2012 he is separated from his first wife, and you get engaged to another woman. After two years comes to know that his first wife was pregnant. At this point, it came to our clinic explaining the story.

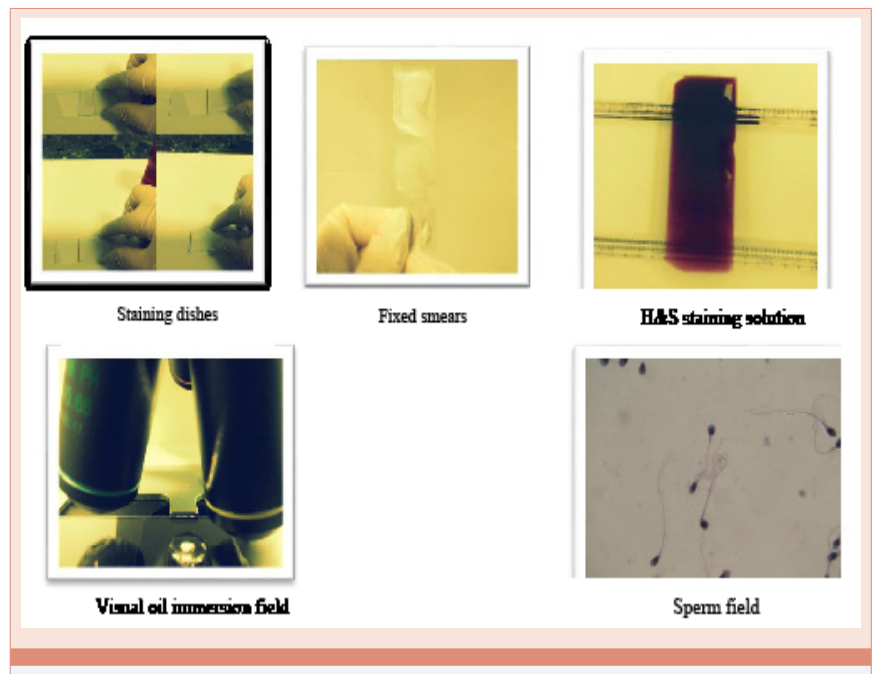

Figure 2: Hematoxylin and Eosin Stain (Medical center -Dani Andrology).

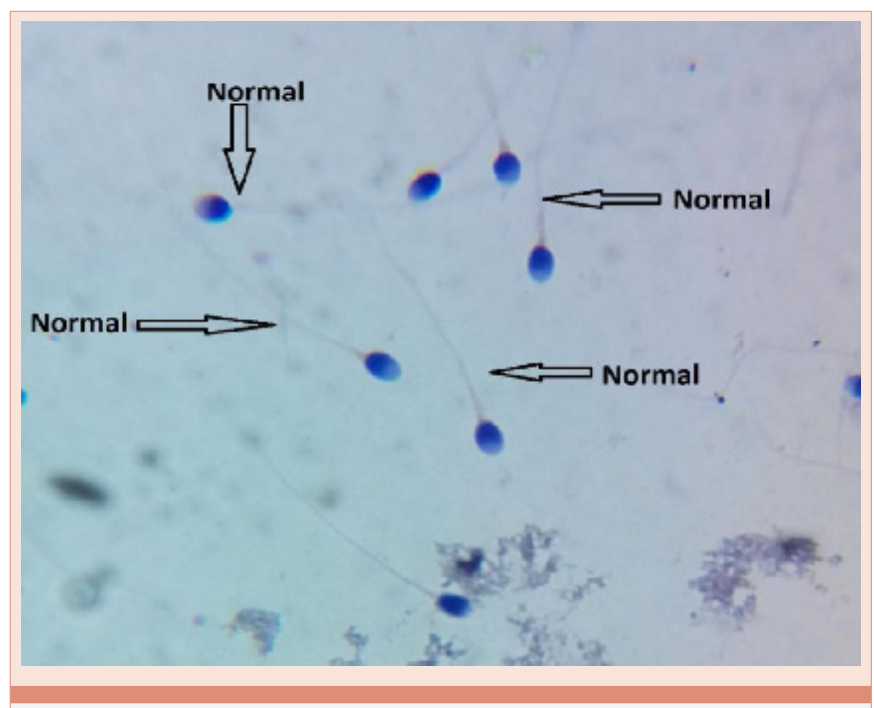

Figure 3: Normal morphology. (Medical center-Dani Andrology). 
After a detailed semen analysis it has been revealed $100 \%$ round headed spermatozoa (Figure 4). Second wife was stimulate using long protocol with $3.75 \mathrm{mg}$ Decapeptyl and 225 IU Menogon for 11 days. From a total of 26 oocytes only 12 have arrived at a fertilization rate. Performed ICSI with assisted oocyte start using a calcium ionophore, was demonstrated 2 Pronuclei in 2 eggs while the other eggs stayed unfertilized. Pregnancy was attained with twins.

The two patients were not pretentious beforehand by cryptorchidism and were not exposed to, chemotherapy agents, pesticides, excessive heat and drugs such as nicotine, alcohol, marijuana and steroids. Checkup investigations revealed a within standard BMI, testicular volume about $4.5 \mathrm{~cm}(\mathrm{~L}), 2.5 \mathrm{~cm}(\mathrm{~W})$, and $2.5 \mathrm{~cm}(\mathrm{H})$, no gynecomastia. Serum testosterone, prolactin LH, FSH levels were normal. This led us to determine that to have a more profound knowledge of the characteristics of our patient infertile pathologies we have to re-consider the cytomorpholgical evaluations.

From these data, there is everything normal, except for the fact that the morphology of the sperm do, was not normal as reported by the other centers but they were round cell sperm. This comes from the fact that many centers do not give much importance to the morphology but she look only at the sperm motility.

Researchers have verified that the acrosome response is essential for fertilization to take place. Remarkably, spermatozoa with acrosome disorder have been actually suggestive of a human sterility condition known as globozoospermia.

"Type I" globozoospermia is illustrated by a key efficient anomaly in the precise division of the acrosome that mirrors an acrosome's enzyme disorder. This sort of spermatozoon is not capable to fertilize the egg and therefore enter the human zona pellucida $[9,10]$. We have also seen that the spermatozoa present structural flaws on the tail's microtubules region, outcome of a so called primary and absolute sterility.

"Type II" globozoospermia is exemplified by a pointed shaped center and a little amount of acrosome. The nucleus is encircled by a huge cytoplasm and is observed merely a derivative diadem signifying degenerative amends. The sterility is originated by pitiable motility but the probability of conception by gonadotropins administration could be amplified.

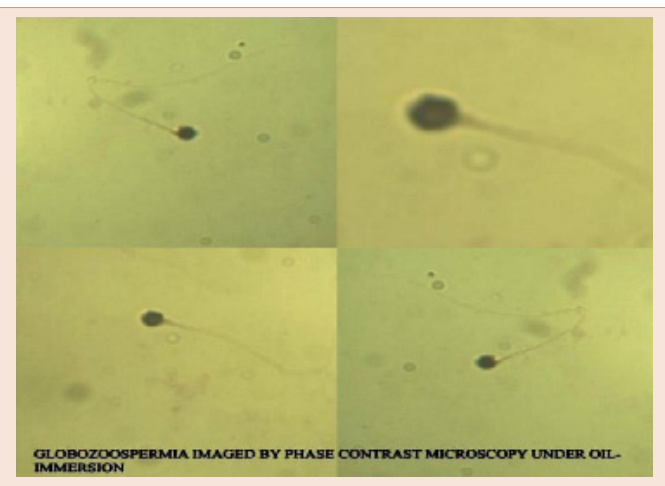

Figure 4: Globozoospermia. Overview imaged by phase contrast microscopy under oil-immersion (Medical center -Dani Andrology).
The DNA information enclosed in the sperm is sheltered from radical oxygen genus by advanced stage of strong chromatin (less condensed DNA tends to be besmirched). It is exposed that globozoospermia as well as other irregular morphologic shapes of human spermatozoa is coupled with DNA strand breaks and abnormal chromatin configures. Though several studies have exposed no momentous amplification in DNA denaturation or sperm DNA disintegration in globozoospermic cases compared productive men [11-13]. Sutovsky et al., in 2001 with the preface of SUTI (sperm- ubiquitin tag immunoassay) practice demonstrated that globozoospermic are extremely ubiquitinated that pointed their injured DNA [14]. In fact, these results suggest that unusual globozoospermic spermatozoa can be the transporter of atypical chromatin as a potential foundation of DNA crumbling. Although, some writers have suggested that the legacy of ancestral globozoospermia may be autosomal recessive [15], autosomal central [16], or polygenic, the precise form of heritage is not apparent.

From a genomic study conducted on a Jordanian family consisting of 5 brothers ( 4 unhealthy and 1 healthy) it was observed that a homozygote removal on chromosome XII of the gene DPY19L2, linked with globozoospermia. Deletion chunks not only the spermatogenesis but also the propagation of germinal cells in meiosis, playing a vital role in acrosome biogenesis. The lack of acrosome entails a failure to conquer zona pellucida barriers. In patient with globozoospermia established the ICSI (intra cytoplasmic sperm injection) is an enormous substitute. Though morphologically usual spermatozoa has a superior rate of success, with the ICSI process, matched with rounded head spermatozoa. The first case of birth by globozoospermia with ICSI was accounted in 1994. Achievement was attained following two successive cycles; from a total of 28 oocytes only 12 have arrived at a fertilization rate.

\section{Discussion}

There is certainly a great evidence of the fact that, a good morphology of the spermatozoa is crucial for the ICSI results. The ICSI introduction has guaranteed an excellent solution for globozoospermia suffering patients in treating their infertility pathologies. A man was diagnosed with globozoospermia with normal sperm count and motility. We performed ICSI with assisted oocyte activation using a calcium ionophore. However, well defined morphological abnormalities such as complete globozoospermia and pin-head have been strongly associated with sterility even in assisted reproductive cycles [1]. Of interest, the outcome of intra- cytoplasmic sperm injection (ICSI) cycles found to be lower when compared with patients with male factor rather than specific morphological abnormalities. 10 Therefore, examination of sperm morphology should not be underestimated in order to define specific sperm abnormalities in male patients with infertility. Genetic related trigger factors (i.e. chromosomal aberrations or genetic mutations) can represent $10-15 \%$ of severe male infertility. Therefore, someone may hypothesize that in patients with globozoospermia, a higher risk by mistakes of the first meiotic division creating aneuploidy ovocitarie after ICSI procedure should be warranted. In conclusion the familial appearance of globozoospermia is extremely rare and the underlying inheritance is not clear. To be concluded although this study has 
not found any $\mathrm{Y}$ chromosome micro deletions in patients with globozoospermia, the exact genetic locus resulting in this pathology requires further study.

\section{References}

1. Dam AH, Feenstra I, Westphal JR, Ramos L, van Golde RJ, et al. (2007) Globozoospermia revisited. Hum Reprod Update 13: 63-75.

2. Menkveld R (1987) An investigation of environmental influences on spermatogenesis and semen parameters. [PhD dissertation (in Afrikaans)]. South Africa: Faculty of Medicine, University of Stellenbosch.

3. Menkveld R, Stander FS, Kotze TJ, Kruger TF, van Zyl JA (1990) The evaluation of morphological characteristics of human spermatozoa according to stricter criteria. Human Reprod5: 586-592.

4. World Health Organisation (2010) WHO Laboratory Manual for the Examination and Processing of Human Semen, 5th ed. Geneva: World Health Organization.

5. World Health Organization (1980) WHO laboratory manual for the examination of human semen and semencervical mucus interaction, 1st edn. Singapore: Press Concern.

6. World Health Organization (1999) WHO laboratory manual for the examination of human semen and spermcervical mucus interaction, 4th edn. Cambridge Cambridge University Press.

7. Oehninger S, Kruger TF, editors (2007) Male Infertility. Diagnosis and Treatment. Oxon: Informa Healthcare. 141: 28.
8. Comhaire F, Schoonjans F, Vermeulen L, De Clercq N (1994) Methodological aspects of sperm morphology evaluation: comparison between strict and liberal criteria. FertilSteril 62: 857-861.

9. Menkveld R, Franken DR, Kruger TF, Oehninger S (1991) Sperm selection capacity of the human zonapellucida. MolReprod Dev 30: 346-352.

10. Liu DY, Baker HW (1992) Morphology of spermatozoa bound to the zonapellucida of human oocytes that failed to fertilize in vitro. J FertilReprod 94: 71-84

11. Vozdova M, Rybar R, Kloudova S, Prinosilova P, Texl P, et al. (2014) Total globozoospermia associated with increased frequency of immature spermatozoa with chromatin defects and aneuploidy: a case report. Andrologia 46: 831- 836.

12. Vicari E, Perdichizzi A, De Palma A, Burrello N, D'Agata R, et al. (2002) Globozoospermia is associated with chromatin structure abnormalities: case report. Hum Reprod 17: 2128-2133

13. Sutovsky P, Terada Y, Schatten G (2001) Ubiquitin-based sperm assay for the diagnosis of male factor infertility. Hum Reprod 16: 250-258.

14. Coetzee K, Windt ML, Menkveld R, Kruger TF, Kitshoff M (2001) An intracytoplasmic sperm injection pregnancy with a globozoospermic male. $J$ Assist Reprod Genet 18: 311-313.

15. Kalahanis J, Rousso D, Kourtis A, Mavromatidis G, Makedos G, et al. (2002) Round-headed spermatozoa in semen specimens from fertile and subfertile men. J Reprod Med 47:489-493.

16. Lanzendorf S, Maloney M, Ackerman S, Acosta A, Hodgen G (1998) Fertilizing potential of acrosomedefective sperm following microsurgical injection into eggs. Gamete Res19: 329-337.

Copyright: (c) 2016 Shkembi E, et al. This is an open-access article distributed under the terms of the Creative Commons Attribution License, which permits unrestricted use, distribution, and reproduction in any medium, provided the original author and source are credited. 